CLINICAL STUDY

\title{
Sick leave for follow-up control in thyroid cancer patients: comparison between stimulation with Thyrogen and thyroid hormone withdrawal
}

\author{
I Borget, C Corone ${ }^{1}, \mathrm{M} \mathrm{Nocaudie}^{2}, \mathrm{M} \mathrm{Allyn}^{3}, \mathrm{~S}$ Iacobelli, M Schlumberger ${ }^{4}$ and G De Pouvourville \\ Department of Health Economics, Institut de Cancérologie Gustave Roussy, 39 rue Camille Desmoulins, 94805 Villejuif, France, ${ }^{1}$ Department of Nuclear \\ Medicine, Centre René Huguenin, Saint-Cloud, France, ${ }^{2}$ Department of Nuclear Medicine, Centre Hospitalier Régional Universitaire de Lille, Lille, France, \\ ${ }^{3}$ Genzyme Corporation, Cambridge, Massachusetts, USA and ${ }^{4}$ Department of Nuclear Medicine and Endocrine Oncology, Institut de Cancérologie Gustave \\ Roussy, Villejuif, France
}

(Correspondence should be addressed to I Borget; Email: borget@igr.fr)

\begin{abstract}
Introduction: The clinical benefits of recombinant human thyroid-stimulating hormone (rhTSH; Thyrogen) are well established as an alternative stimulation procedure to thyroid hormone withdrawal in the diagnostic follow-up of thyroid cancer patients. By avoiding periods of hypothyroidism, patients do not suffer from a decreased quality of life and keep their ability to work. This study compared the frequency, the duration and the cost of sick leave for follow-up control between rhTSH and withdrawal. Methods: The study population consisted of patients with thyroid carcinoma first treated by thyroidectomy and radioiodine ablation. Patients were recruited at their control visit between October 2004 and May 2006 in three hospitals, both prospectively and retrospectively. Collection data consisted of patient information, job characteristics and duration of sick leave during the month before and the month after control. The valuation of sick leave used the friction cost method.

Results: Among the 306 patients included, 292 (95\%) completed the entire questionnaire. The mean age was 46.7 years. Among the 194 active patients, patients treated with rhTSH, when compared with patients treated by withdrawal, were less likely to require sick leave (11 vs 33\%; $P=0.001)$. The mean duration of sick leave was shorter (3.1 vs 11.2 days; $P=0.002$ ) and indirect costs due to absenteeism accounted for $€ 454 \pm 1673$ vs $€ 1537 \pm 2899$ for withdrawal stimulation.

Conclusion: For active patients, rhTSH treatment reduced the length and the cost of sick leave by 8.1 days and $€ 1083$ per control respectively, when compared with withdrawal treatment.
\end{abstract}

European Journal of Endocrinology 156 531-538

\section{Introduction}

In Europe, 26000 new cases of thyroid cancer are diagnosed each year (1). Differentiated thyroid carcinoma represents more than $80 \%$ of all thyroid cancers and frequently affects working-age subjects. Its favourable long-term prognosis with a 10 -year survival of $90-95 \%$ (2) is the result of an effective primary therapy, which consists of a total thyroidectomy followed by radioiodine ablation where there is a significant risk of persistent disease $(3,4)$. Patients are then treated with $\mathrm{L}$-thyroxine (LT4). Follow-up control is designed to detect persistent or recurrent disease with neck ultrasonography and measurement of serum thyroglobulin $(\mathrm{Tg})$, and, when required, with whole body scan (WBS) (3-5). Serum Tg determination is more sensitive for the detection of recurrent disease when performed following TSH stimulation than on thyroid hormone treatment (6).

TSH stimulation was historically achieved by thyroid hormone withdrawal (THW) for 3-5 weeks when patients suffered from symptoms of hypothyroidism. Recombinant human TSH (rhTSH, Thyrogen, Genzyme Therapeutics, Cambridge, MA, USA) was approved in Europe in 2000 as an alternative stimulation procedure to THW. It allows patients to remain euthyroid on thyroid hormone therapy, and Tg determination has a sensitivity for disease detection similar to that obtained following THW (6-8).

The greatest impairment in the quality of life (QoL) of thyroid cancer patients occurred during THW which induces a hypothyroid state (9-13). Even though the design and the scales of QoL of these studies were quite different, it appears that patients were mainly suffering from decreased psychological and physical well-being, low vitality and lowered ability to perform normal daily activities. Moreover, symptoms related to cancer and/or hypothyroidism can cause a temporary inability to work. Sick leave is an indirect measure of morbidity in the working population. Schroeder et al. showed that $74 \%$ of patients had difficulties in performing work after 
withdrawal, when compared with $18 \%$ of those on LT4 and $21 \%$ of those undergoing rhTSH stimulation (13). The quantitative effects of hypothyroidism on patient's productivity have been described only in two studies that reported a median of 11 days of absence from work during the period of hypothyroidism induced by withdrawal $(12,14)$. However, these studies were not modelled to directly compare the duration of sick leave and its economic impact according to the method of TSH stimulation, and they did not directly estimate sick leave for patients receiving rhTSH.

Costs due to loss of productivity, also referred to as indirect costs, are an important part of the total costs of cancer. In 2000, the National Institute of Health, reporting disease-specific estimated costs of illness, showed that the cost of cancer was estimated to be $\$ 96$ billion, of which $\$ 69$ billion were related to indirect costs. The incidence of thyroid carcinoma is increasing (15) and it affects working-age patients. Since the follow-up should be long term and this cancer is associated with a low mortality, the number of patients in follow-up is large (estimated to be 400000 in Europe). This cancer may have an important and increasing economic impact on health, as the inability to work may result in an important economic loss. Indirect costs are also an important component of costeffectiveness studies published in the domain of thyroid cancer treatment, as demonstrated by Mernagh et al. (16), who used published data to estimate loss of productivity costs in their assessment of the incremental cost-per-QALY of rhTSH in the course of the treatment of thyroid cancer.

Unfortunately, there are no published data from a direct comparison of the impact on work loss between patients stimulated by withdrawal versus rhTSH. The aim of this study is to perform such a direct comparison and evaluate the impact of hormone withdrawal versus rhTSH on sick-leave frequency, duration and economic impact by a direct assessment of sick leave periods for follow-up controls in France.

\section{Materials and methods}

\section{Patients}

This study included patients with papillary or follicular thyroid carcinoma, without known distant metastasis, who had initially been treated by total thyroidectomy and radioiodine ablation following withdrawal. Patients were recruited both retrospectively and prospectively between October 2004 and May 2006 in three French hospitals: Institut Gustave Roussy (IGR, Villejuif, France), Centre René Huguenin (CRH, Saint-Cloud) and Centre Hospitalier Regional and Universitaire de Lille (CHRU Lille, France). A retrospective collection was required to ensure the inclusion of a sufficiently large number of patients in the withdrawal group, since at the time of the study there was a very limited number of specialised nuclear medicine centres which still practised withdrawal.

Patients included in the study underwent a control either 6-12 months after initial treatment or later during follow-up. This control was performed after TSH stimulation obtained either by a withdrawal of thyroid hormone treatment or during thyroid hormone treatment by i.m. injections of recombinant human TSH (Thyrogen, Genzyme Therapeutics, $0.9 \mathrm{mg}$ i.m. for two consecutive days). Patients were not randomised, because randomisation was not considered acceptable by the local Ethics Committee since the benefits of rhTSH in terms of QoL are already well established $(7,8,13)$. The study was then performed in three different centres, of which one used exclusively rhTSH, one predominantly rhTSH and one predominantly THW. Depending on local practice, followup included a physical examination, a serum sampling for $\mathrm{Tg}$ and TSH determinations and a neck ultrasonography or a diagnostic ${ }^{131}$ I WBS.

\section{Questionnaire}

The questionnaire was divided into three parts. The first part included a patient consent form, socio-demographic data, information about the thyroid cancer treatment (date of thyroidectomy and the number of radioiodine treatments), the method of stimulation and examinations performed during this control. It also included employment status, nature of job and the type of insurance coverage (French National Insurance coverage or other). The employment status of patients was classified into five pre-defined categories: remunerated activity, job seeker, pensioner, without professional activity (e.g. housewife) and other conditions (student or other). Active patients refer to those who had a remunerated activity or were job seekers. The second and the third parts of the questionnaire were administered only to active patients; the patients had to report the number of days out of work occurring during the month before and the month after the control, respectively (Fig. 1).

The administration of the questionnaire was different according to the recruitment of patients. For patients recruited prospectively (from April 2005 to May 2006), the physician obtained the patient's informed consent and collected the first and the second parts of the questionnaire during the control. Then, the patients sent back the third part of the questionnaire after completing the number of days of sick leave occurring during the 30 days following control. Patients recruited retrospectively had a control visit between October 2004 and March 2005 and were contacted in April 2005. The maximum recall period was then 6 months in order to limit memory bias. Medical and sociodemographic data were extracted from the medical record. A document was sent to these patients by postal service including a presentation of the study, a consent 


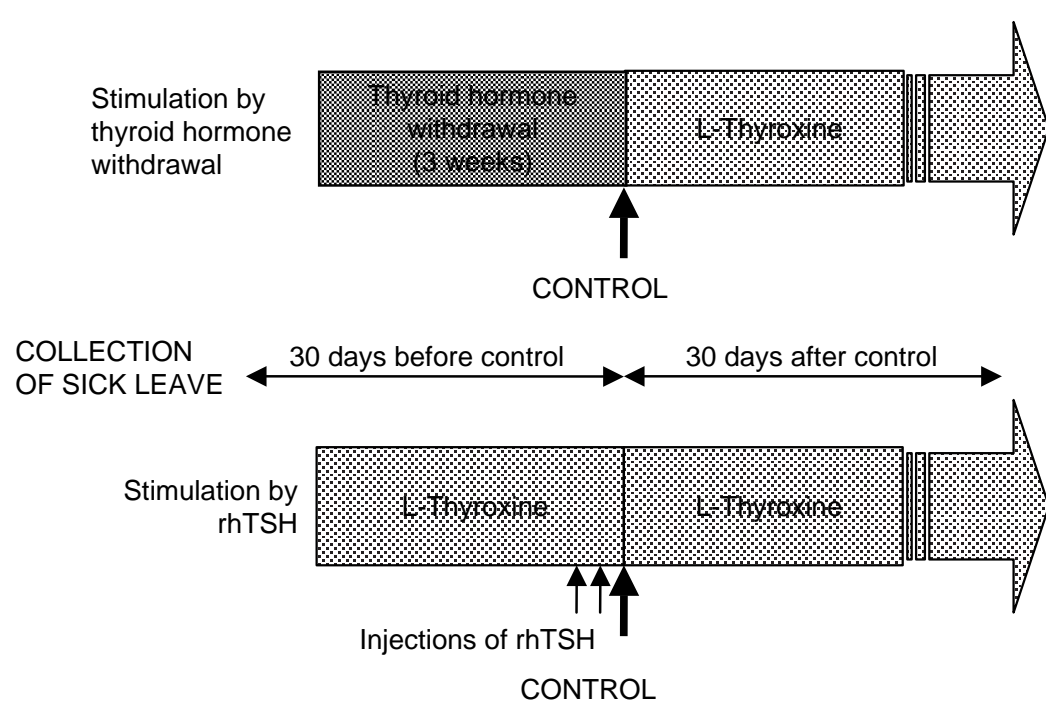

Figure 1 Description of time of TSH stimulation and time of collection of sick leave.

form and a questionnaire. Patients had to complete all three parts of the questionnaire. For both cases, a phone call was made by the coordinator of the study (IB) if the questionnaire was not returned in due time.

\section{Valuation of sick leave}

Budgetary and economic approaches were used to assess the costs of sick leave.

The budgetary approach relates to the budget impact for the National Sickness Fund of monetary compensation given to patients to partially offset their loss of income. In France, compensations are paid by this Fund from day 4 onwards. A reduction in sick leave compensation is a budget saving for the payer, which may offset an extra cost of treatment. The value of 1 day of sick leave compensation was calculated by dividing the total amount of compensation paid in year 2003 by the total number of sick leave days (data provided by the National Health Accounts) and was estimated to be $€ 27$.

The economic consequences evaluated the impact of a disease and its treatment on the productivity of active patients and on the production of wealth at a national level, thus using a societal perspective. We used the friction cost approach: the assumption is that disease causes losses in production, but this loss is restricted to an adjustment period needed to adapt to the absenteeism, the so-called friction period (FC) (17-20). Since the impact on productivity and production is limited to this adjusted 'FC', absenteeism does not translate in a oneto-one decrease in production. The FC approach uses an elasticity of production per working day of 0.8 : 1 day out of work translates into a reduction of 0.8 days of lost production of wealth (17). The value of lost production to consider is not the Gross National Product (GNP), but the National Value Added, which subtracts the value of intermediary goods that are used in the production process from the GNP, multiplied by the number of lost working days in patients with remunerated activity only (not job seekers).

\section{Statistical methods}

Quantitative data are expressed as means and s.D. and qualitative data are expressed as percentages. Significant differences between both groups were tested with a $\chi^{2}$-test.

The duration and the cost of sick leave were expressed as means instead of medians because more than $50 \%$ of active patients did not have any sick leave (median would be equal to 0 in the two groups) and referred to active patients only. Since the duration and costs of sick leave violate normality assumptions, significant differences in sick leave duration and cost were assessed with a Kruskal-Wallis non-parametric test.

A logistic regression analysis using the individual characteristics of active patients was performed to identify factors associated with the probability to have sick leave and to control for selection bias due to the use of a nonrandomised comparison. It aimed to determine whether eight parameters (sex, age, type of recruitment of patient, type of insurance coverage, number of radioiodine treatments, interval between thyroidectomy and control, ${ }^{131}$ I administration for WBS and method of stimulation) influenced the probability of having sick leave during the period. Results are expressed in odds ratios (OR).

All the statistical analyses were performed with SAS software version 9.1.

\section{Results}

Three hundred and six patients were included in the study. Of these, $232(76 \%)$ patients were included prospectively. Among the 306 patients, 5 patients did not return their questionnaire, 8 patients returned an 
Table 1 Initial characteristics of the patients.

\begin{tabular}{lcccc}
\hline & $\begin{array}{c}\text { Stimulation by } \\
\text { withdrawal }\end{array}$ & $\begin{array}{c}\text { Stimulation by } \\
\text { rhTSH }\end{array}$ & Comparison & All patients \\
\hline Number of patients $(n)$ & 119 & 173 & & 292 \\
Number of patients included & 67 & 161 & 228 \\
$\quad \begin{array}{l}\text { prospectively }(n) \\
\text { Age (years) }\end{array}$ & $93(78 \%)$ & $123(71 \%)$ & $P=0.17$ & $216(74 \%)$ \\
Number of radioiodine treatments & $47.7 \pm 13.6(18-80)$ & $46.0 \pm 12.8(18-75)$ & $P=0.26$ & $46.7 \pm 13.2(18-80)$ \\
Interval between thyroidectomy & $1.1 \pm 0.3(1-3)$ & $1.2 \pm 0.6(1-5)$ & $P=0.02$ & $1.1 \pm 0.4(1-5)$ \\
$\quad$ and control (years) & $1.5 \pm 1.6(0.6-13.9)$ & $3.4 \pm 3.7(0.4-20.7)$ & $P<0.0001$ & $2.6 \pm 3.1(0.4-20.7)$ \\
Interval between the last radioiodine & $0.9 \pm 0.7(0.4-6.1)$ & $2.9 \pm 3.3(0.2-19.9)$ & $P<0.0001$ & $2.1 \pm 2.8(0.2-19.9)$ \\
$\quad$ treatment and control (years) & & & & \\
\hline
\end{tabular}

Mean \pm s.D. (range).

incomplete questionnaire (without the third part) and 1 patient refused to participate. The analysis was based on the 292 patients with a completed questionnaire, leading to a global response rate of $95 \%$.

In total, stimulation was achieved in 173 patients by rhTSH injections and in 119 patients by withdrawal. Baseline demographic characteristics are reported in Table 1. Two hundred and sixteen patients (74\%) were female. The mean age was $46.7 \pm 13.2$ years. With respect to age and sex ratio, there was no significant difference between the two groups of stimulation, between the 292 patients who completed the questionnaire and the 14 patients whose questionnaire was incomplete or absent, and between the 228 patients included prospectively and the 64 patients included retrospectively.

Patients had received a mean of 1.1 radioiodine treatments (one treatment in 262 patients, two in 23 patients, three in 6 patients and five in 1 patient). Patients underwent surgery 2.6 years before control and received the last radioiodine treatment 2.1 years before control. The mean intervals of time between initial surgery and control and between the last radioiodine treatment and control were significantly longer in patients stimulated by rhTSH than in those stimulated by withdrawal.

Investigations performed were different among the three centres (Table 2). Patients who had their control at IGR or CRH were mainly treated by rhTSH, whereas patients from Lille were mainly stimulated by THW.
All patients underwent serum $\mathrm{Tg}$ determination and almost all also a TSH determination. Diagnostic WBS was performed in all patients who had undergone withdrawal and in $42 \%$ of patients who had undergone rhTSH stimulation. Neck ultrasonography was routinely performed only in the two centres which preferentially used rhTSH stimulation.

The professional status of the patients is presented in Table 3. The cohort comprised 194 (66\%) active patients, composed of 176 patients with a remunerated activity and 18 job seekers. The other 98 patients were retired (50), had no professional activity (36), were students (7), were handicapped or disabled (3), or were living in foreign countries and did not have sick leave compensation from the French National Health System (2). There was no significant difference in the working status between the two groups of stimulation $\left(\chi^{2}=3.41 ; P=0.49\right)$. Two hundred and fifty nine $(89 \%)$ patients were affiliated to the French National Insurance coverage for Salaried Workers. The other 33 patients were affiliated to the Farmers Fund (6), the SelfEmployed Fund (7), different state employee insurance (9), the students' fund (3) and others funds (8). There was no significant difference in the insurance coverage between the two groups of stimulation $(P=0.18)$. There was no statistical difference in terms of age, sex, employment status, rate of activity or type of insurance coverage between the three centres.

The results of frequency and the duration of sick leave are described only for the 194 active patients (Table 4).

Table 2 Methods of stimulation and examinations performed by centre.

\begin{tabular}{lccr}
\hline & IGR $(n=82$ patients $)$ & CRH $(n=87$ patients $)$ & Lille $(n=123$ patients $)$ \\
\hline $\begin{array}{l}\text { Method of stimulation }(n) \\
\quad \text { Withdrawal }\end{array}$ & 0 & 5 & 114 \\
$\quad$ rhTSH & 82 & 82 & 9 \\
Type of recruitment $(n)$ & & & \\
$\quad$ Prospective & 76 & 87 & 65 \\
Examinations performed (\%) & & 100 & 100 \\
$\quad$ Thyroglobulin determination & 100 & 99 & 2 \\
$\quad$ Neck ultrasonography & 100 & 71 & 100 \\
$\quad$ Whole body scan & 7 & 92 & 100 \\
$\quad$ TSH determination & 98 & & \\
\hline
\end{tabular}


Table 3 Professional status of the patients.

\begin{tabular}{lccc}
\hline & Withdrawal $(n=119)$ & rhTSH $(n=173)$ & All patients $(n=292)$ \\
\hline Employment status $n(\%)$ & & & \\
Remunerated activity & $66(55 \%)$ & $110(64 \%)$ & $176(60 \%)$ \\
Jobless & $7(6 \%)$ & $11(6 \%)$ & $18(6 \%)$ \\
Pensioner & $21(18 \%)$ & $29(17 \%)$ & $50(17 \%)$ \\
Without professional activity & $19(16 \%)$ & $17(10 \%)$ & $36(13 \%)$ \\
Other & $6(5 \%)$ & $6(6 \%)$ & $12(4 \%)$ \\
Active patient $n(\%)$ & $73(61 \%)$ & $121(70 \%)$ & $194(66 \%)$ \\
Type of insurance coverage $n(\%)$ & $102(86 \%)$ & $157(91 \%)$ & $259(89 \%)$ \\
French National Insurance coverage & $17(14 \%)$ & $16(9 \%)$ & $33(11 \%)$ \\
Other regimen & & \\
\hline
\end{tabular}

Before control, the number of active patients who did not require sick leave was higher in the rhTSH group when compared with the withdrawal group (92 and $75 \%$ respectively, $P=0.001)$. Mean sick leave duration was shorter in active patients treated by rhTSH than in those treated by withdrawal (1.4 vs 5.0 days respectively, $P=0.0015$; Table 4).

After the control, the number of patients who did not require sick leave was higher after preparation by rhTSH than in patients treated by withdrawal (90 and $68 \%$ respectively, $P=0.0001)$. Mean sick leave duration after control was shorter in active patients stimulated by rhTSH than in patients treated by withdrawal (1.7 vs 6.2 respectively, $P=0.0001$; Table 4 ).

From 30 days before to 30 days after control, the number of patients who did not need sick leave during the entire period was higher in patients treated by rhTSH than in those stimulated by withdrawal (89 and $67 \%$ respectively, $P=0.0001)$. Mean sick leave duration was shorter in active patients treated by rhTSH than in those treated by withdrawal (3.1 vs 11.2 days respectively, $P=0.001$; Table 4). The rhTSH stimulation allowed a reduction of 8.1 days of sick leave length per active patient. There was no significant difference in terms of duration of sick leave between the patients included prospectively and the patients included retrospectively.

The logistic regression analysis concerning the effect of eight individual characteristics on the probability to have sick leave showed that women were 4.3 times more at risk than men (Table 5), but this probability did not depend on age at the time of the study, number of years since thyroidectomy and number of radioiodine treatments. The probability to have sick leave was lower in patients who did not belong to the French National Insurance coverage, but it was higher in patients recruited prospectively $(\mathrm{OR}=2.1, \mathrm{CI}=0.78-5.74)$ and was 1.6 times higher $(\mathrm{CI}=0.46-5.38)$ in patients who underwent a WBS, but these differences were not statistically significant. In active patients treated by rhTSH, the mean duration of sick leave with WBS was not significantly different from that of patients who did not undergo a WBS (3.5 vs 2.8 days respectively, $P=0.74)$. Indeed, the risk to have sick leave was 3.6 times higher in patients treated by THW and this was statistically different from the risk to have sick leave in patients treated by rhTSH.

In patients stimulated by withdrawal only, the probability to have sick leave in patients included prospectively was 2.6 times higher than in patients included retrospectively, but this coefficient was not significant at the 0.05 level. Moreover, mean global sick leave duration was longer in active patients recruited prospectively than in active patients recruited retrospectively (13.2 vs 9.2 days respectively), but there was no significant difference.

\section{Valuation of sick leave}

The mean cost in terms of loss of productivity in 2005 amounted to $€ 193$ per working day in patients with a remunerated activity (by considering 5 working days per week). The valuation of indirect costs of absenteeism by the method of friction cost was $€ 1537 \pm 2899$ for active patients treated by withdrawal vs $€ 454 \pm 1673$

Table 4 Mean duration of sick leave.

\begin{tabular}{lccc}
\hline & Treated by withdrawal & Treated by rhTSH & $\begin{array}{c}\text { Comparison with non-parametric } \\
\text { test (Kruskal-Wallis, } P \text { ) }\end{array}$ \\
\hline Duration of sick leave (days) & & & \\
$\quad$ Before control & $5.0 \pm 10.2(0-30)$ & $1.4 \pm 6.0(0-30)$ & 0.0015 \\
$\quad$ After control & $6.2 \pm 10.7(0-30)$ & $1.7 \pm 6.4(0-30)$ & 0.0001 \\
$\quad$ All the period & $11.2 \pm 19.9(0-60)$ & $3.1 \pm 11.5(0-60)$ & 0.0001 \\
Valuation of sick leave $(€)$ & $275 \pm 503,(0-1539)$ & $74 \pm 288,(0-1539)$ & $<0.0001$ \\
$\quad$ Budgetary impact & $1537 \pm 2899,(0-9640)$ & $454 \pm 1673,(0-9061)$ & 0.0003 \\
$\quad$ Economic impact & & \\
\hline
\end{tabular}


Table 5 Influence of parameters associated with the risk to have sick leave.

\begin{tabular}{lc}
\hline Parameters & $\begin{array}{c}\text { Odds ratio } \\
\text { (95\% confidence interval) }\end{array}$ \\
\hline Sex & 1 \\
$\quad$ Men & \\
$\quad$ Women & $4.32(1.40 ; 13.35)^{\star}$ \\
Age & $1.00(0.97 ; 1.05)$ \\
Type of recruitment of patient & 1 \\
$\quad$ Retrospective & $2.12(0.78 ; 5.74)$ \\
$\quad$ Prospective & $0.86(0.69 ; 1.07)$ \\
Interval between thyroidectomy and control & $0.59(0.14 ; 2.50)$ \\
Number of radioiodine treatments & 1 \\
Type of insurance coverage & \\
$\quad$ French National Insurance coverage & $0.38(0.09 ; 1.56)$ \\
$\quad$ Others funds & 1 \\
Whole body scan (WBS) & $1.55(0.45 ; 5.38)$ \\
$\quad$ No WBS & 1 \\
$\quad$ WBS & $3.58(1.30 ; 9.90)^{*}$ \\
Stimulation method & \\
$\quad$ rTSH & \\
$\quad$ Withdrawal & \\
\hline &
\end{tabular}

for active patients treated by rhTSH $(P=0.0003)$. The mean difference of absenteeism cost according to the method of stimulation is estimated to be $€ 1083$ per follow-up control in active patients (Table 4).

The average sick leave compensation per day was equal to $€ 27$. From the perspective of the payer, the mean financial impact of sick leave was higher in active patients treated by withdrawal than in those treated by rhTSH, with $€ 275$ vs 74 respectively $(P<0.0001$; Table 4). The money saved with stimulation by rhTSH is then estimated to be $€ 201$ per follow-up control in active patients.

\section{Discussion}

This survey was designed to compare the frequency, the duration and the economic impact of sick leave periods according to the two methods of stimulation, withdrawal of thyroid hormone treatment or recombinant human TSH, for the diagnosis of recurrent or persistent disease. In France, there are no national statistics about the number of lost working days for a given disease. Thus, an alternative method is to design an ad hoc survey and to collect data from patients in selected centres. To our knowledge, this is the largest cohort study of thyroid cancer patients in which the impact on sick leave periods according to the method of stimulation has been investigated through a direct comparison. The study was multicentric and the high response rate to the questionnaire $(95 \%$ of completed questionnaires) facilitated the generalisation of our results to thyroid cancer patients. Our findings are of the same order of magnitude with the results of previous studies that estimated the duration of sick leave caused by hypothyroid symptoms. In our study, the mean duration of sick leave for a short-term period of hypothyroidism is 11.2 days in active patients, which is similar to the Dutch results (14). In Germany, Luster et al. observed a median sick leave duration of 11 days in case of withdrawal (12), but their study had some limitations: response rate was lower $(61 \%)$, leading to a potential selection bias of patients, and the duration of sick leave in patients treated by rhTSH was estimated through an expert panel. Since sick leave compensation systems may be different from one country to another, this may be coincidental, but confirms that the sick leave dimension is not to be neglected in the economic balance.

One limit of the present study was the absence of randomisation between the two protocols of stimulation. Randomisation was not considered acceptable by the local Ethics Committee because the benefits of rhTSH in terms of QoL are already well established $(7,8$, 13). Patients included in the study underwent a control either 6-12 months after initial treatment or later during follow-up and the characteristics of the patients depended on the patient recruitment of each centre, leading to a possible selection bias, for example on the general health status of patients. But both groups of stimulation were similar in terms of age, sex and working status. The results showed that the initial treatment of patients stimulated by rhTSH had occurred at greater distance in time from follow-up than for patients stimulated by withdrawal. It could be argued that distance from initial treatment is correlated with a better health status of patients, thus requiring fewer sick leaves. Conversely, patients in the rhTSH group had received more radioiodine treatments than patients stimulated by withdrawal and the results of logistic regression showed that the risk of having sick leave in active patients was not influenced by either parameter. Finally, we also controlled for a potential bias because of the retrospective inclusion of patients, particularly in the withdrawal group. We limited the bias using a short period of recall. Nevertheless, considering the impact of withdrawal on the QoL, it could be expected that patients who stopped working may have overestimated the duration of sick leave, whereas patients who had very short sick leaves may have omitted to report them. When comparing both retrospective and prospective data in the withdrawal group, analysis did not confirm these assumptions, suggesting a limited bias.

Another difference was that radioiodine WBS was performed in all patients in the withdrawal group, but in only $42 \%$ of patients in the rhTSH group. The risk of having sick leave in patients who underwent a WBS was higher than the risk associated with patients who did not have WBS, but it did not reach statistical difference. The variable 'centre' was not entered as an independent variable in the logistic regression analysis because it was strongly correlated to the variables 'WBS' and 'stimulation method', and we preferred to test the effect of these variables rather than the effect of centre. 
According to the logistic regression analysis, sex and method of stimulation were the only parameters that significantly increased the risk of having sick leave.

Days lost from work are a common proxy for morbidity, particularly in thyroid cancer patients who are working-age patients and free of disease at follow-up control. Hypothyroidism can have a major impact on work schedules among thyroid cancer survivors $(9,13)$. Our study demonstrates and quantifies the benefits of using rhTSH: patients required shorter periods of sick leave than those stimulated by withdrawal. The reduction of the duration of sick leave with rhTSH stimulation allows patients to return to work more rapidly than after withdrawal.

All the patients included in this study, whatever the stimulation method used for the control, had previously experienced THW, especially for thyroid remnants ablation. The positive subjective feeling in patients receiving rhTSH and, in the opposite, the negative feeling in patients resubmitted to THW may increase the difference in sick leave duration between the two methods of stimulation. However, the logistic regression analysis showed that the numbers of radioiodine treatments (and the number of episodes of THW) did not have any effect on the probability of having sick leave.

An interesting finding of our study is that the impact of hypothyroidism on sick leave is actually greater after than before diagnostic control, even though LT4 therapy has been resumed. This underlines that the debilitating effects of hypothyroidism persist for a longer time period than the weeks of pre-testing withdrawal from LT4.

The valuation of sick leave considered both the budgetary and the economic consequences of lost working days. In active patients, stimulation by rhTSH allows money saving of $€ 201$ (budgetary impact, from the National Health System perspective), whereas the reduction of indirect costs in terms of productivity loss is estimated to be $€ 1083$ per follow-up control (economic impact, from the society perspective). The difference in magnitude of costs between the two approaches can be easily explained. The National Health System compensates only and partially the loss of wages for patients. In France, the acquisition cost of rhTSH is $€ 762$ per control. Thus, from the point of view of the payer, the reduction in the level of sick leave allows to offset a fraction of the drug cost $(201 / 762=26 \%)$, notwithstanding the other benefits of rhTSH. In France, the same Fund pays for both health care and sick leave, and it should be able to monitor effective reductions in compensations paid due to rhTSH. This may be more difficult in other countries where sick leave and health care are paid by different funds, and thus induce a restricted use of rhTSH due to budgetary constraints. From the perspective of society, the economic valuation of the number of lost working days is based on the loss of productivity of active patients and on the production of wealth at a national level and is much greater than the budget impact to the payer.

\section{Conclusion}

When compared with withdrawal stimulation, rhTSH stimulation demonstrated similar diagnostic accuracy in the detection of recurrence or residual disease in patients with thyroid cancer. By avoiding hypothyroidism, patients do not suffer from a decreased QoL and maintain their ability to work, which reduces cost for the payer and for society.

\section{Acknowledgements}

Funded by an unrestricted grant from Genzyme Corporation.

\section{References}

1 Boyle P \& Ferlay J. Cancer incidence and mortality in Europe, 2004. Annals of Oncology 200516 481-488.

2 Mazzaferri EL. An overview of the management of papillary and follicular thyroid carcinoma. Thyroid 19999 421-427.

3 Pacini F, Schlumberger M, Dralle H, Elisei R, Smit JW \& Wiersinga $W$. European consensus for the management of patients with differentiated thyroid carcinoma of the follicular epithelium. European Journal of Endocrinology 2006154 787-803.

4 Cooper DS, Doherty GM, Haugen BR, Kloos RT, Lee SL, Mandel SJ, Mazzaferri EL, Mciver B, Sherman SI \& Tuttle RM. Management guidelines for patients with thyroid nodules and differentiated thyroid cancer. Thyroid $2006 \mathbf{1 6} 109-142$.

5 Schlumberger M, Pacini F, Wiersinga WM, Toft A, Smit JW, Sanchez Franco F, Lind P, Limbert E, Jarzab B, Jamar F, Duntas L, Cohen $O$ \& Berg G. Follow-up and management of differentiated thyroid carcinoma: a European perspective in clinical practice. European Journal of Endocrinology 2004151 539-548.

6 Eustatia-Rutten CF, Smit JW, Romijn JA, Van Der Kleij-Corssmit EP, Pereira a M, Stokkel MP \& Kievit J. Diagnostic value of serum thyroglobulin. Clinical Endocrinology 200461 61-74.

7 Haugen BR, Pacini F, Reiners C, Schlumberger M, Ladenson PW, Sherman SI, Cooper DS, Graham KE, Braverman LE, Skarulis MC, Davies TF, Degroot LJ, Mazzaferri EL, Daniels GH, Ross DS, Luster M, Samuels MH, Becker DV, Maxon HR III, Cavalieri RR, Spencer CA, Mcellin K, Weintraub BD \& Ridgway EC. A comparison of recombinant human thyrotropin and thyroid hormone withdrawal for the detection of thyroid remnant or cancer. Journal of Clinical Endocrinology and Metabolism $1999 \mathbf{8 4}$ $3877-3885$.

8 Ladenson PW, Braverman LE, Mazzaferri EL, Brucker-Davis F, Cooper DS, Garber JR, Wondisford FE, Davies TF, Degroot LJ, Daniels GH, Ross DS \& Weintraub BD. Comparison of administration of recombinant human thyrotropin with withdrawal of thyroid hormone for radioactive iodine scanning in patients with thyroid carcinoma. New England Journal of Medicine 1997337 888-896.

9 Dow KH, Ferrell BR \& Anello C. Balancing demands of cancer surveillance among survivors of thyroid cancer. Cancer Practice $19975289-295$.

10 Davids T, Witterick IJ, Eski S, Walfish PG \& Freeman JL. Threeweek thyroxine withdrawal: a thyroid-specific quality of life study. Laryngoscope 2006116 250-253.

11 Dow KH, Ferrell BR \& Anello C. Quality-of-life changes in patients with thyroid cancer after withdrawal of thyroid hormone therapy. Thyroid 19977 613-619.

12 Luster M, Felbinger R, Luster M, Felbinger R, Dietlein M \& Reiners C. Thyroid hormone withdrawal in patients with differentiated thyroid carcinoma: a one hundred thirty-patient 
pilot survey on consequences of hypothyroidism and a pharmacoeconomic comparison to recombinant thyrotropin administration. Thyroid 200515 1147-1155.

13 Schroeder PR, Haugen BR, Pacini F, Reiners C, Schlumberger M, Sherman SI, Cooper DS, Schuff KG, Braverman LE, Skarulis MC, Davies TF, Mazzaferri EL, Daniels GH, Ross DS, Luster M, Samuels MH, Weintraub BD, Ridgway EC \& Ladenson PW. A comparison of short-term changes in health-related quality of life in thyroid carcinoma patients undergoing diagnostic evaluation with recombinant human thyrotropin compared with thyroid hormone withdrawal. Journal of Clinical Endocrinology and Metabolism 200691 878-884.

14 Nijhuis TF, Van Weperen W \& De Klerk JMH. Costs associated with the withdrawal of thyroid hormone suppression therapy during the follow-up treatment of well-differenciated thyroid cancer. Tijdschrift voor Nucleaire Genneskunde 199921 98-100.

15 Colonna M, Grosclaude P, Remontet L, Schvartz C, Mace-Lesech J, Velten M, Guizard A, Tretarre B, Buemi aV, Arveux P \& Esteve J. Incidence of thyroid cancer in adults recorded by French cancer registries (1978-1997). European Journal of Cancer 200238 $1762-1768$.
16 Mernagh P, Campbell S, Dietlein M, Luster M, Mazzaferri E \& Weston aR. Cost-effectiveness of using recombinant human TSH prior to radioiodine ablation for thyroid cancer, compared with treating patients in a hypothyroid state: the German perspective. European Journal of Endocrinology 2006155 405-414.

17 Koopmanschap MA, Rutten FF, Van Ineveld BM \& Van Roijen L. The friction cost method for measuring indirect costs of disease. Journal of Health Economics 199514 171-189.

18 Hodgson TA. Costs of illness in cost-effectiveness analysis. A review of the methodology. Pharmacoeconomics 19946 536-552.

19 Liljas B. How to calculate indirect costs in economic evaluations. Pharmacoeconomics 199813 1-7.

20 Brouwer WB \& Koopmanschap MA. The friction-cost method: replacement for nothing and leisure for free? Pharmacoeconomics 200523 105-111.

Received 4 December 2006

Accepted 7 February 2007 